\title{
Spin-dependent tunneling between individual superconducting bound states
}

\author{
Haonan Huang, ${ }^{1}$ Jacob Senkpiel, ${ }^{1}$ Ciprian Padurariu $\odot{ }^{2},{ }^{2}$ Robert Drost, ${ }^{1}$ Alberto Villas, ${ }^{3}$ Raffael L. Klees $\odot{ }^{4}$, \\ Alfredo Levy Yeyati, ${ }^{3}$ Juan Carlos Cuevas ${ }^{0},{ }^{3}$ Björn Kubala, ${ }^{2}$ Joachim Ankerhold $\odot,{ }^{2}$ Klaus Kern, ${ }^{1,5}$ and Christian R. Ast ${ }^{1}{ }^{1 *}$ \\ ${ }^{1}$ Max-Planck-Institut für Festkörperforschung, Heisenbergstraße 1, 70569 Stuttgart, Germany \\ ${ }^{2}$ Institut für Komplexe Quantensysteme and IQST, Universität Ulm, Albert-Einstein-Allee 11, 89069 Ulm, Germany \\ ${ }^{3}$ Departamento de Física Teórica de la Materia Condensada and Condensed Matter Physics Center (IFIMAC), \\ Universidad Autónoma de Madrid, 28049 Madrid, Spain \\ ${ }^{4}$ Fachbereich Physik, Universität Konstanz, 78457 Konstanz, Germany \\ ${ }^{5}$ Institut de Physique, Ecole Polytechnique Fédérale de Lausanne, 1015 Lausanne, Switzerland
}

(Received 17 November 2020; accepted 24 May 2021; published 8 July 2021)

\begin{abstract}
Magnetic impurities on superconductors induce discrete bound levels inside the superconducting gap, known as Yu-Shiba-Rusinov (YSR) states. YSR levels are fully spin polarized such that the tunneling between YSR states depends on their relative spin orientation. Here, we use scanning tunneling spectroscopy to resolve the spin dynamics in the tunneling process between two YSR states by experimentally extracting the angle between the spins. To this end, we exploit the ratio of thermally activated and direct spectral features in the measurement to directly extract the relative spin orientation between the two YSR states. We find freely rotating spins down to $7 \mathrm{mK}$, indicating a purely paramagnetic nature of the impurities. Such a noncollinear spin alignment is essential not only for producing Majorana bound states but also as an outlook manipulating and moving the Majorana state onto the tip.
\end{abstract}

DOI: 10.1103/PhysRevResearch.3.L032008

Magnetic impurities on superconductors give rise to a wealth of phenomena due to the subtle interplay between pairing processes of conduction electrons and their exchange interaction with the impurity [1-6]. As a consequence, single spin-nondegenerate bound states inside the superconducting gap may emerge [7-16]. A fundamental understanding of the role of spin in the coupling between such states is highly relevant [17], for example, in the context of creating Majorana bound states at the ends of a chain of Yu-Shiba-Rusinov (YSR) states $[18,19]$. Aside from the conventional quasiparticle hopping from one site to the next, pair creation from the superconducting condensate can also couple two neighboring sites in a chain of YSR states. To reach the topological regime in such a chain, the coexistence of both types of coupling processes between neighboring sites is indispensable [20,21]. The spin provides a vital component selecting between the two coupling processes. Conventional hopping is favored when the spins of two sites are aligned, while pair creation is favored when neighboring spins are anti-aligned.

In past experiments, we have observed end states on magnetic atom chains on superconductors using scanning tunneling microscopy (STM) $[2,18,19]$, but the two tunneling

\footnotetext{
*Corresponding author: c.ast@fkf.mpg.de
}

Published by the American Physical Society under the terms of the Creative Commons Attribution 4.0 International license. Further distribution of this work must maintain attribution to the author(s) and the published article's title, journal citation, and DOI. Open access publication funded by the Max Planck Society. processes between neighboring sites in the chain could not be individually accessed. To separate the two tunneling processes, control over the energy detuning between adjacent sites is needed. For this purpose, instead of using the tip as an external sensor [19,22], measuring a YSR state with a functionalized YSR tip turns the tunnel junction into an internal probe of the coupling between two YSR sites.

Here, we use STM [23] to couple a YSR state on the vanadium tip and an intrinsic YSR state on the $\mathrm{V}(100)$ surface, where we independently resolve direct and thermally activated Shiba-Shiba tunneling [Figs. 1(a)-1(c)]. We first show experimentally from the spin blockade of a characteristic Andreev reflection that an individual state is spin nondegenerate. Measuring the two tunnel processes between two spin-nondegenerate superconducting bound states, we find that both direct and thermal processes are present for all measured impurities. Their coexistence excludes the possibility that the impurity spins are collinear, fulfilling the requirement for use in topological chains. The measured relative strength of the two tunnel processes enables us to extract the angle $\theta$ of the relative spin orientation. Tracing the relative spin orientation from $1 \mathrm{~K}$ to $7 \mathrm{mK}$, we find $\theta=90^{\circ}$ in all measurements, indicating freely rotating spins of the YSR states in tip and sample with no detectable anisotropy. At the end, we draw an analogy between Shiba-Shiba tunneling and the coupling of neighboring states in a YSR chain.

When both tip and sample exhibit a YSR state, the tunneling between these discrete levels can be observed in the current-voltage $I(V)$ characteristic as isolated spectral features [24]. At $10 \mathrm{mK}$, we only observe current peaks at bias voltages indicated by blue arrows labeled $d^{ \pm}$in Fig. 1(d), 


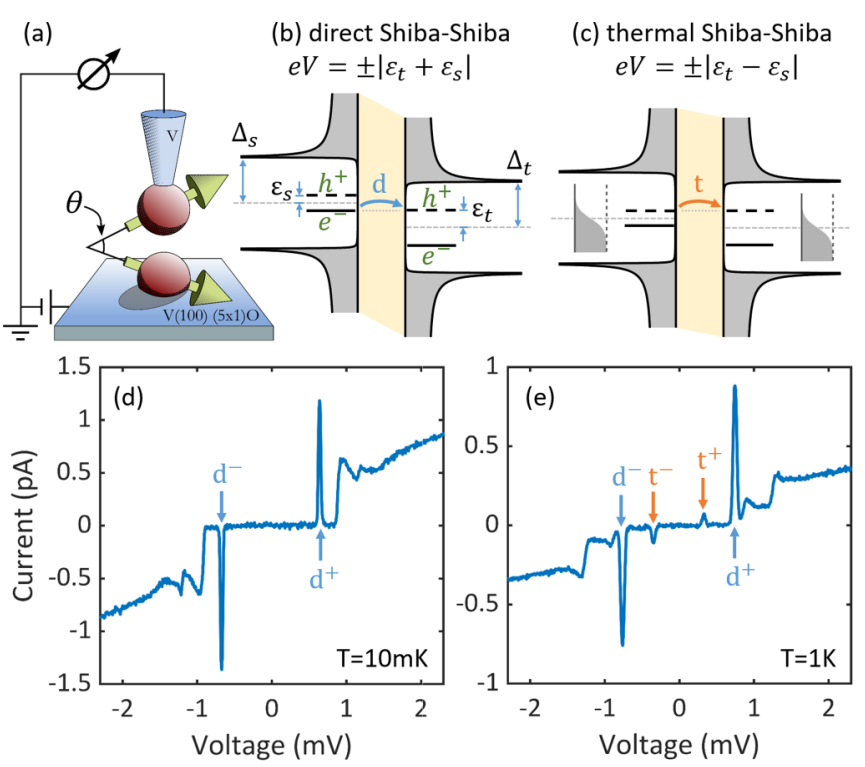

FIG. 1. Direct Shiba-Shiba tunneling and thermal Shiba-Shiba tunneling. (a) Schematics of the experimental setup realizing tunneling between Yu-Shiba-Rusinov (YSR) states using a scanning tunneling microscope (STM), with $\theta$ denoting the angle between the two spins. Tunneling processes of (b) direct and (c) thermal Shiba-Shiba tunneling. In the direct process, the $e^{-}$and $h^{+}$parts of the two YSR states are aligned by the bias voltage. In the thermal process, the two $e^{-}$or two $h^{+}$parts are aligned. $\Delta_{s, t}$ are the superconducting gap parameters of the sample and tip, respectively, with both $\sim 760 \mu \mathrm{eV}$ for vanadium. Typical $I(V)$ spectra of Shiba-Shiba tunneling at (d) $10 \mathrm{mK}$ and (e) $1 \mathrm{~K}$. Blue arrows $\left(d^{ \pm}\right)$indicate direct Shiba-Shiba peaks, and orange arrows $\left(t^{ \pm}\right)$indicate thermal Shiba-Shiba peaks.

which we refer to as direct Shiba-Shiba tunneling. This process is found at a bias voltage $V$ of the sum of two YSR energies $e V= \pm\left|\epsilon_{t}+\epsilon_{s}\right|$ ( $e$ is the electron charge, and $\epsilon_{t, s}$ are YSR energies for the tip and sample, respectively) and can in principle occur at $0 \mathrm{~K}$. It is plotted schematically in Fig. 1(b).

At higher temperature $(1 \mathrm{~K})$, however, the YSR state may get thermally excited, such that thermally activated tunneling becomes possible. Figure 1(c) schematically shows the tunneling process involving a thermally excited YSR state. Additional current peaks appear at the bias voltage $e V=$ $\pm\left|\epsilon_{t}-\epsilon_{s}\right|$ [see Fig. 1(e) indicated by orange arrows and labeled $\left.t^{ \pm}\right]$. We call this process thermal Shiba-Shiba tunneling because it is exponentially suppressed toward zero temperature. The intensity of the thermal Shiba-Shiba peaks is smaller than the direct Shiba-Shiba peak due to the small thermal Boltzmann factor at $1 \mathrm{~K}$. Due to their appearance at distinct bias voltages, the direct and thermal Shiba-Shiba processes can be separately addressed in a single measured $I(V)$ spectrum. While the general dynamics of the tunneling processes of Shiba-Shiba tunneling has been discussed recently [24], the role of the spin in the transport process is more subtle but plays a defining part in the coupling between YSR states.

Conventional models describe YSR states as spinnondegenerate states, i.e., $100 \%$ spin polarized [8,26], but not necessarily with a preferred axis. Still, it should be experi-
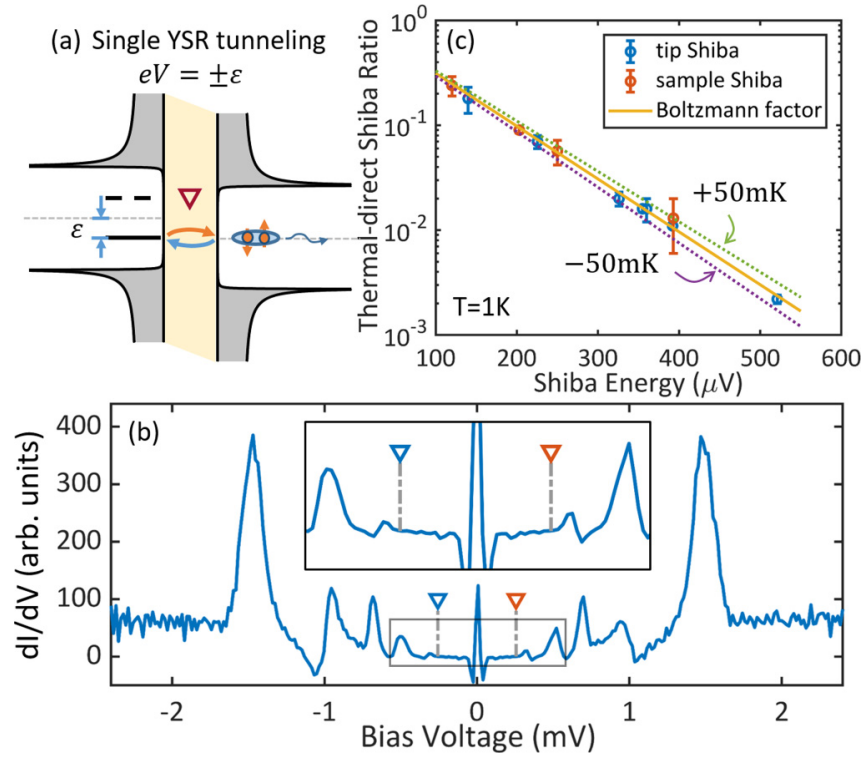

FIG. 2. Properties of single Yu-Shiba-Rusinov (YSR) states and conventional YSR-BCS (Bardeen-Cooper-Schrieffer) tunneling. (a) A family of multiple Andreev reflection processes (MARs) connecting the levels of the same YSR state (the schematic here shows only the lowest order process). Due to full spin polarization of the YSR state, this family of MARs is spin forbidden. (b) A typical $d I / d V$ spectrum involving one YSR state tunneling into a clean superconductor at high conductance (here, $\sim 0.16 G_{0}$, where $G_{0}=2 e^{2} / h$ is the conductance quantum) to reveal MARs. Detailed peak assignment can be found in the Supplemental Material [25]. The expected position for the lowest order MARs in (a) is labeled with symbol $\nabla$, where no peak is seen confirming the spin blockade. (c) Statistics of the thermal-direct ratio of the conventional YSR-BCS peak (in the low conductance regime) of various YSR impurities (seven tip YSR and four sample YSR) with different YSR energy measured at $1 \mathrm{~K}$. Notice that, here, only a single YSR state is involved for each data point. All data points fall on top of the prediction of the Boltzmann factor at $1 \mathrm{~K}$ within a narrow window $( \pm 50 \mathrm{mK})$.

mentally verified that an alleged YSR resonance actually is spin polarized [27,28]. Without resorting to magnetic fields, this can be checked by looking at the presence or absence of the single Andreev reflection process involving the same bound state [29], as shown in Fig. 2(a) for only one YSR state in the tunnel junction (either tip or sample). The absence of such a spectral feature at a bias voltage of $e V= \pm \epsilon$ indicates that the bound state is a spin-nondegenerate YSR state [29]. A high conductance spectrum of a single YSR state in the tunnel junction featuring multiple Andreev reflection peaks is shown in Fig. 2(b). At the expected bias voltage, the respective single Andreev process is absent in the measurement, i.e., there is no spectral feature at the voltage indicated by the symbol $\nabla$, which confirms the spin nondegeneracy of the YSR state (for the assignment of all observed peaks and other details, see the Supplemental Material [25]).

To understand the influence of spin on the Shiba-Shiba peak intensities, we first consider the limiting case of the YSR states being aligned either parallel $\left(\theta=0^{\circ}\right)$ or antiparallel $\left(\theta=180^{\circ}\right)$, as shown in Figs. 3(a)-3(d). If the spins of the corresponding parts of the YSR states are parallel, direct 

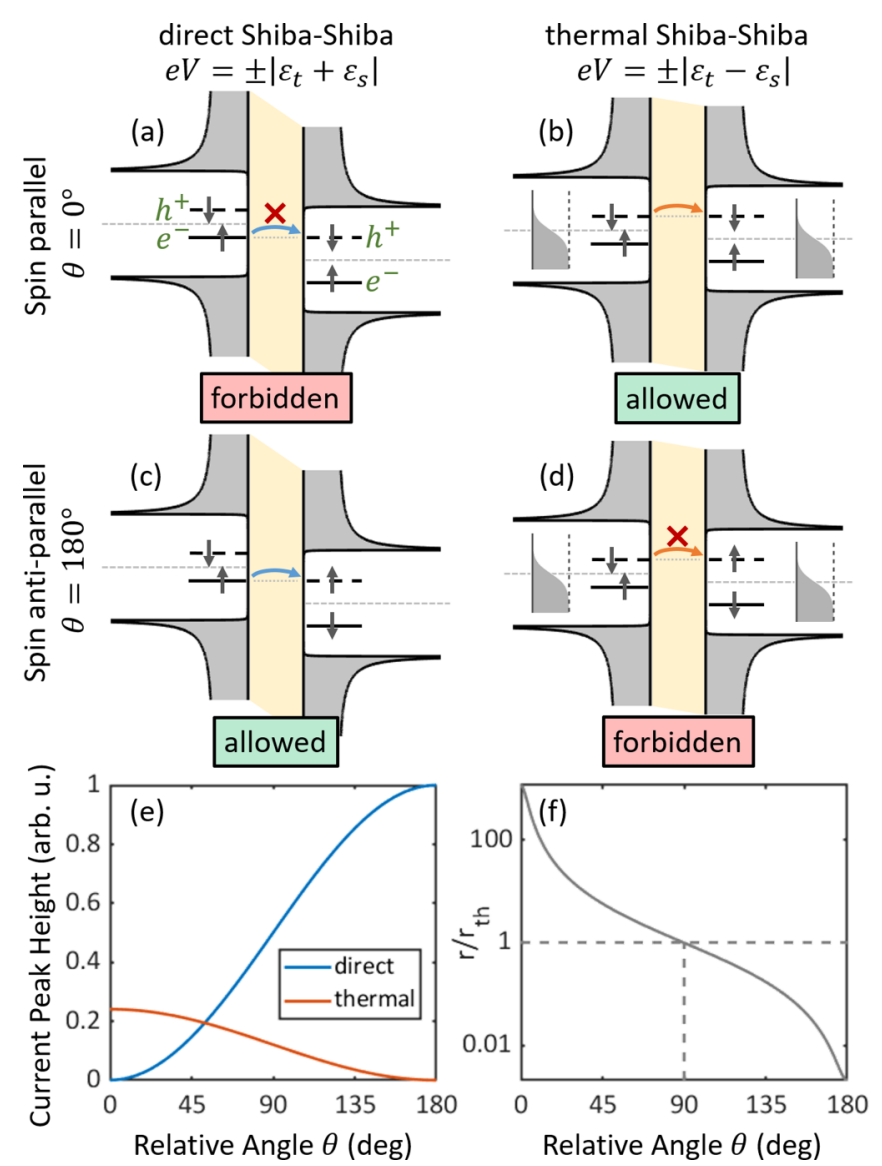

FIG. 3. Theory showing thermal-direct Shiba-Shiba ratio as a measure of the relative spin orientation $\theta$. (a) and (b) The tip and sample Yu-Shiba-Rusinov (YSR) states have parallel spins $\left(\theta=0^{\circ}\right)$. Note that the $e^{-}$and $h^{+}$parts of the same YSR state always have opposite spins [26-28]. Here, the direct Shiba-Shiba process is forbidden, while thermal Shiba-Shiba tunneling is allowed due to spin conservation during tunneling. (c) and (d) The tip and sample YSR states have opposite spins $\left(\theta=180^{\circ}\right)$. Here, the direct Shiba-Shiba process is allowed, while thermal Shiba-Shiba tunneling is forbidden. (e) The continuous dependency of the direct and thermal Shiba-Shiba peak intensity on the relative spin angle. (f) The universal dependency of $r / r_{\text {th }}$ on the relative spin angle.

Shiba-Shiba tunneling is forbidden [Fig. 3(a)]. This is due to spin conservation in the tunneling process and because the electron $\left(e^{-}\right)$and hole $\left(h^{+}\right)$parts, which participate in the tunneling process, have opposite spins. The thermal Shiba-Shiba process, however, is allowed because no spin blockade exists [Fig. 3(b)] for tunneling between two hole parts due to parallel spins.

If the spins of the YSR states are antiparallel, the situation is reversed. Using similar arguments as above, direct ShibaShiba tunneling is now allowed [Fig. 3(c)], while thermal Shiba-Shiba tunneling is forbidden [Fig. 3(d)] (for a detailed discussion, see the Supplemental Material [25]) [8,26,27].

Observing both direct and thermal Shiba-Shiba peaks in Fig. 1(e) directly leads to the conclusion that the spins in the measurement can neither be completely parallel nor completely antiparallel. Using the standard expression for the tunneling current explicitly including the spin [25]

$$
I(V)=\sum_{\sigma}\left[I_{\sigma \sigma}(V) \cos ^{2} \frac{\theta}{2}+I_{\sigma \bar{\sigma}}(V) \sin ^{2} \frac{\theta}{2}\right],
$$

where $\sigma=\uparrow, \downarrow$, and $\bar{\sigma}=\downarrow, \uparrow$, the $I(V)$ spectra can be simulated expanding the above discussion to arbitrary angles between the spins of the two YSR states $\left(0^{\circ}<\theta<180^{\circ}\right.$; for details, see the Supplemental Material [25]). The result is shown in Fig. 3(e). The Shiba-Shiba peak intensities evolve smoothly between the extreme cases of parallel and antiparallel orientations, confirming the limiting cases discussed before. In the measurement, we find two peaks for each direct and thermal process (one at positive and one at negative bias voltages). To account for both peaks in the analysis, we average between them using the geometric mean which eliminates the electron-hole asymmetry and the absolute intensity of the peaks, so that the resulting ratio $r$ only depends on the angle $\theta$ between the spins of the two YSR states as well as the Boltzmann factors of the two YSR states

$r \stackrel{\text { def }}{=} \sqrt{\frac{p_{t^{+} p_{t^{-}}}}{p_{d^{+}} p_{d^{-}}}}=\cot ^{2}\left(\frac{\theta}{2}\right)\left|\exp \left(-\frac{\epsilon_{\mathrm{s}}}{k_{\mathrm{B}} T}\right)-\exp \left(-\frac{\epsilon_{\mathrm{t}}}{k_{\mathrm{B}} T}\right)\right|$,

where $T$ is the temperature, $k_{\mathrm{B}}$ is the Boltzmann constant, and $p_{i}$ with $i=d^{ \pm}, t^{ \pm}$is the intensity of the corresponding Shiba-Shiba peak. Further, we define a parameter $r_{\text {th }}$ as the difference of the two Boltzmann factors, which are experimentally accessible, such that we find

$$
\frac{r}{r_{\mathrm{th}}}=\cot ^{2}\left(\frac{\theta}{2}\right)
$$

Note that, here, it is possible to use either current peak area or peak height because the width of direct and thermal Shiba-Shiba is the same (for details, see the Supplemental Material [25]).

The predicted evolution of $r / r_{\text {th }}$ is plotted in Fig. 3(f), which maps a unique angle for every ratio. If $r / r_{\text {th }} \rightarrow \infty$, the spins are parallel; if $r / r_{\text {th }} \rightarrow 0$, spins are antiparallel. Equation (3) presents a universal curve that is independent of the details of each YSR state. Because the Shiba-Shiba peaks are typically well separated in the measured spectrum, both $r$ and $r_{\text {th }}$ in Eq. (3) can be precisely derived from directly measurable quantities in a single measurement, such that the angle $\theta$ can be experimentally determined. It is this ability to independently resolve the relative strength of two different Shiba-Shiba tunneling processes in the $I(V)$ spectrum, which enables an all-electrical measurement of the relative spin-orientation in our experiment - a striking difference to conventional spin-valve experiments, where a polarizing magnetic field is used to rotate (or flip) the relative orientation.

To extract the Boltzmann factors, we consider experimentally the thermal-to-direct ratio of the YSR state tunneling into the continuum [30]. The statistics of such measurements at $1 \mathrm{~K}$ are plotted in Fig. 2(c) (for details, see the Supplemental Material [25]). All Boltzmann ratios for both tip and sample YSR states lie on a line corresponding to $1 \pm 0.05 \mathrm{~K}$. This 

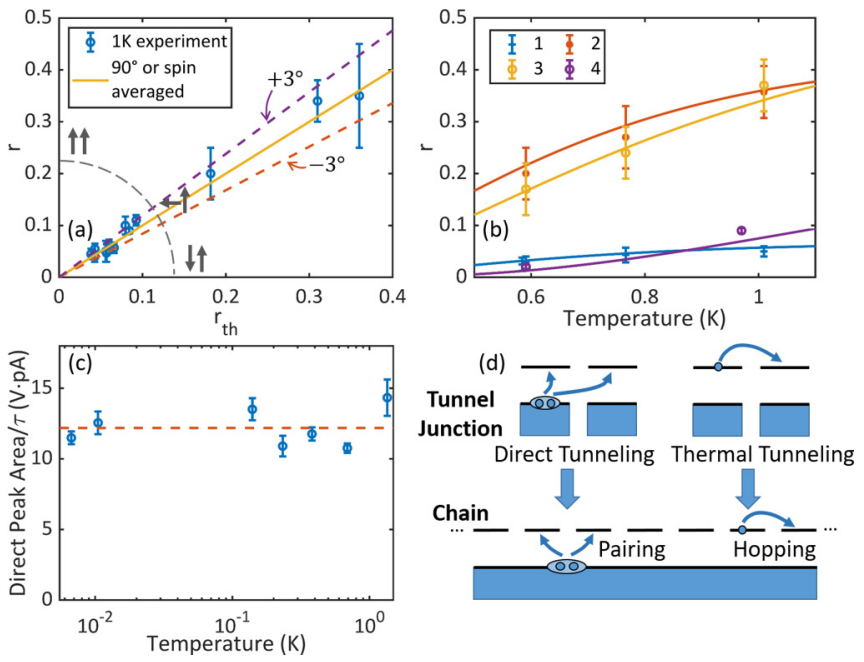

FIG. 4. Temperature dependency measurements. (a) Statistics of the thermal-direct Shiba-Shiba ratio $r$ (of peak current) measured at $1 \mathrm{~K}$, plotted against the difference of the Boltzmann factor $r_{\text {th }}$ of tip and sample Shiba states. There are 13 data points resulting from different combinations of seven different YSR tips and seven different sample YSR impurities. All data points lie on the identity line within a small error bar, indicating a spin averaging effect. (b) The temperature dependence of thermal-direct Shiba-Shiba ratio $r$ of selected examples with different YSR energies (data points) agreeing with the prediction from the difference of the Boltzmann factor $r_{\text {th }}$ (curves of corresponding color). (c) The temperature dependence of the direct Shiba-Shiba current peak area normalized by the transmission $\tau=G_{N} / G_{0}$, which remains nearly constant, with no indication of spin freezing down to $7 \mathrm{mK}$. (d) The direct and thermal tunneling between two YSR states constitute the fundamental interaction mechanism of the formation of a chain of magnetic impurities on a superconductor. The coexistence of both processes is crucial for the chain to host topologically nontrivial end states.

agrees with our thermometer reading and also confirms that we have only one pair of YSR states inside the gap [30].

We have collected the experimental parameters $r$ and $r_{\text {th }}$ from a number of different Shiba-Shiba systems in the linear tunneling regime using intrinsic defects on the V(100) surface and a $\mathrm{V}$ tip at $1 \mathrm{~K}$. Note that, in the tunneling regime, the twostate system is minimally disturbed as higher order tunneling processes are suppressed. In Fig. 4(a), we plot the thermaldirect Shiba-Shiba ratio $r$ against $r_{\text {th }}$. Interestingly, for all Shiba-Shiba systems, the data points lie on the identity line $\left(r=r_{\text {th }}\right)$ within a small error interval. According to Eq. (3), this means that the relative angle between the two YSR spins is $\theta=90^{\circ} \pm 3^{\circ}$. Considering that every Shiba-Shiba system in Fig. 4(a) shows the same angle $\theta$, it is unlikely that these angles are time independent. Instead, we are measuring the average angle of a time-dependent spin orientation. The tunneling time is short compared to any motion of the spins, such that every tunneling event will observe a static snapshot of the system, so that the measured current is a time average over many independent tunneling events. Therefore, we propose that the spins are freely rotating, giving an average effect $\langle r\rangle=r_{\text {th }}$, where \langle\rangle indicates averaging over an angle isotropically distributed over the unit sphere (for more details, see the Supplemental Material [25]). Note that, although the spin is freely rotating, the electron and hole parts of the same YSR state have opposite spin direction at any time.

A temperature of $1 \mathrm{~K}$ may still be too high for a magnetic system to develop an easy axis [31], which could explain the observed isotropy. We, therefore, trace the parameters $r$ and $r_{\text {th }}$ as a function of temperature down to $0.6 \mathrm{~K}$. Figure 4(b) shows the thermal-direct Shiba-Shiba peak ratio $r$ for four different Shiba-Shiba systems between 0.6 and $1 \mathrm{~K}$. Upon cooling, the data points for the ratio $r$ nicely follow the calculation from Eq. (2) (solid lines), assuming an angle $\theta=90^{\circ}$. This indicates that $r=r_{\text {th }}$ with a still freely rotating spin scenario down to $0.6 \mathrm{~K}$.

If we cool further down $<0.6 \mathrm{~K}$, the ratio becomes more difficult to access because of the exponential suppression of thermal Shiba-Shiba peak. However, recalling Fig. 3(e), we note that the direct Shiba-Shiba peak intensity by itself critically depends on the relative spin angle $\theta$. To eliminate the conductance dependency as well as temperature effects on the YSR lifetime and environmental broadening [24,32-35], we use the peak area normalized by the conductance (see the Supplemental Material for details [25]). Figure 4(c) shows a typical dataset of the normalized direct Shiba-Shiba peak area from $1 \mathrm{~K}$ down to $7 \mathrm{mK}$, showing no significant change over two decades of temperature range. These measurements are consistent with a relative spin orientation rotating isotropically down to $7 \mathrm{mK}$ without any anisotropy energy $\left(k_{\mathrm{B}} T<0.6 \mu \mathrm{eV}\right)$. We find that, for YSR states, the relative orientation of the spins determines whether direct or thermal tunneling is possible (cf. Fig. 3). This is different from the spin valve effect in normal conducting tunnel junctions, where an anti-alignment suppresses the tunneling current but does not favor a different transport channel.

It is tempting to draw an analogy between the two coupled YSR states in our experiment and a link in a chain of YSR states [2]. The two types of coupling processes, direct and thermal Shiba-Shiba tunneling, are counterparts of the pair creation and hopping terms in the Kitaev Hamiltonian [see Fig. 4(d)] [20,24,25]. In this analogy, Majorana end states require the coexistence of both coupling processes, which is guaranteed by the noncollinearity of the neighboring impurity spins. We surmise that the relative spin orientation between YSR states is equally important in the chain as in the tunnel junction. One difference, however, is that, in our experiment, the two impurities are placed on different superconducting substrates relatively far away compared to the atomic chain. This rules out magnetic interactions via the substrate, which have been suggested to induce helical order in Kitaev chains [36-42]. Nevertheless, as an outlook, our results suggest an intriguing experiment to probe the edge of a magnetic impurity chain by a YSR tip. The tip YSR state will couple to the state at the edge of the chain by direct and thermal Shiba-Shiba tunneling and extend the chain by one site. The Majorana end state will move from the substrate onto the tip.

In summary, we propose the ratio between the thermal and direct Shiba-Shiba tunneling peaks as a direct and unambiguous all-electrical probe for the relative spin orientation between YSR states. We conclude that, for the Shiba-Shiba systems consisting of intrinsic YSR impurities on V(100) and YSR states on the vanadium tip, the YSR states derive from freely rotating quantum spin- $1 / 2$ impurities from 
$1 \mathrm{~K}$ down to $7 \mathrm{mK}$. The spin plays a defining role in the transport between spin-nondegenerate superconducting bound states selecting between hopping and pairing mechanisms (i.e., direct and thermal Shiba-Shiba tunneling). Additionally, the analogy between the Shiba-Shiba junction and a chain link in a YSR chain attributes a critical role to the spin for producing Majorana end states. Further, a YSR tip in close proximity to a Majorana end state may induce a topological phase transition allowing the Majorana end state to be manipulated by the tip. Going beyond the immediate consequences, a Shiba-Shiba junction may also provide the basis for a minimal source to inject triplet Cooper pairs into a superconductor [43].
We would like to acknowledge fruitful discussions with Gianluca Rastelli and Wolfgang Belzig. This letter was funded in part by the European Research Council Consolidator Grant AbsoluteSpin (Grant No. 681164) and by the Center for Integrated Quantum Science and Technology. J.A. acknowledges funding from the German Research Foundation (DFG) under Grant No. AN336/11-1. A.V., A.L.Y., and J.C.C. acknowledge funding from the Spanish Ministry of Economy and Competitiveness (Grant No. FIS2017-84057-P and No. FIS2017-84860-R), from the "María de Maeztu" Programme for Units of Excellence in R\&D (No. MDM-2014-0377). R.L.K. is grateful for funding provided by the DFG through Grant No. RA 2810/1.
[1] S. H. Pan, E. W. Hudson, K. M. Lang, H. Eisaki, S. Uchida, and J. C. Davis, Imaging the effects of individual zinc impurity atoms on superconductivity in $\mathrm{Bi}_{2} \mathrm{Sr}_{2} \mathrm{CaCu}_{2} \mathrm{O}_{8+\delta}$, Nature (London) 403, 746 (2000).

[2] S. Nadj-Perge, I. K. Drozdov, J. Li, H. Chen, S. Jeon, J. Seo, A. H. MacDonald, B. A. Bernevig, and A. Yazdani, Observation of Majorana fermions in ferromagnetic atomic chains on a superconductor, Science 346, 602 (2014).

[3] G. C. Ménard, S. Guissart, C. Brun, R. T. Leriche, M. Trif, F. Debontridder, D. Demaille, D. Roditchev, P. Simon, and T. Cren, Two-dimensional topological superconductivity in $\mathrm{Pb} / \mathrm{Co} / \mathrm{Si}$ (111), Nat. Commun. 8, 2040 (2017).

[4] S. Kezilebieke, R. Zitko, M. Dvorak, T. Ojanen, and P. Liljeroth, Observation of coexistence of Yu-Shiba-Rusinov states and spin-flip excitations, Nano Lett. 19, 4614 (2019).

[5] A. Odobesko, D. Di Sante, A. Kowalski, S. Wilfert, F. Friedrich, R. Thomale, G. Sangiovanni, and M. Bode, Observation of tunable single-atom Yu-Shiba-Rusinov states, Phys. Rev. B 102, 174504 (2020).

[6] S. Kezilebieke, M. N. Huda, V. Vano, M. Aapro, S. C. Ganguli, O. J. Silveira, S. Głodzik, A. S. Foster, T. Ojanen, and P. Liljeroth, Topological superconductivity in a designer ferromagnet-superconductor van der Waals heterostructure, Nature (London) 588, 424 (2020).

[7] L. Yu, Bound state in superconductors with paramagnetic impurities, Acta Phys. Sin 21, 75 (1965).

[8] H. Shiba, Classical spins in superconductors, Prog. Theor. Phys. 40, 435 (1968).

[9] A. I. Rusinov, Superconductivity near a paramagnetic impurity, JETP Lett. 9, 85 (1969).

[10] A. Yazdani, B. A. Jones, C. P. Lutz, M. F. Crommie, and D. M. Eigler, Probing the local effects of magnetic impurities on superconductivity, Science 275, 1767 (1997).

[11] S.-H. Ji, T. Zhang, Y.-S. Fu, X. Chen, X.-C. Ma, J. Li, W.-H. Duan, J.-F. Jia, and Q.-K. Xue, High-Resolution Scanning Tunneling Spectroscopy of Magnetic Impurity Induced Bound States in the Superconducting Gap of Pb Thin Films, Phys. Rev. Lett. 100, 226801 (2008).

[12] K. J. Franke, G. Schulze, and J. I. Pascual, Competition of superconducting phenomena and Kondo screening at the nanoscale, Science 332, 940 (2011).

[13] N. Hatter, B. W. Heinrich, D. Rolf, and K. J. Franke, Scaling of Yu-Shiba-Rusinov energies in the weak-coupling Kondo regime, Nat. Commun. 8, 2016 (2017).
[14] L. Malavolti, M. Briganti, M. Hänze, G. Serrano, I. Cimatti, G. McMurtrie, E. Otero, P. Ohresser, F. Totti, M. Mannini, R. Sessoli, and S. Loth, Tunable spin-superconductor coupling of spin 1/2 vanadyl phthalocyanine molecules, Nano Lett. 18, 7955 (2018).

[15] J. Senkpiel, C. Rubio-Verdú, M. Etzkorn, R. Drost, L. M. Schoop, S. Dambach, C. Padurariu, B. Kubala, J. Ankerhold, C. R. Ast, and K. Kern, Robustness of Yu-Shiba-Rusinov resonances in the presence of a complex superconducting order parameter, Phys. Rev. B 100, 014502 (2019).

[16] H. Huang, R. Drost, J. Senkpiel, C. Padurariu, B. Kubala, A. Levy Yeyati, J. C. Cuevas, J. Ankerhold, K. Kern, and C. R. Ast, Quantum phase transitions and the role of impurity-substrate hybridization in Yu-Shiba-Rusinov states, Commun. Phys. 3, 199 (2020).

[17] J. C. Estrada Saldana, A. Vekris, R. Zitko, G. Steffensen, P. Krogstrup, J. Paaske, K. Grove-Rasmussen, and J. Nygard, Two-impurity Yu-Shiba-Rusinov states in coupled quantum dots, Phys. Rev. B 102, 195143 (2020).

[18] H. Kim, A. Palacio-Morales, T. Posske, L. Rózsa, K. Palotás, L. Szunyogh, M. Thorwart, and R. Wiesendanger, Toward tailoring Majorana bound states in artificially constructed magnetic atom chains on elemental superconductors, Sci. Adv. 4, eaar5251 (2018).

[19] A. Kamlapure, L. Cornils, J. Wiebe, and R. Wiesendanger, Engineering the spin couplings in atomically crafted spin chains on an elemental superconductor, Nat. Commun. 9, 3253 (2018).

[20] A. Y. Kitaev, Unpaired Majorana fermions in quantum wires, Phys. Usp. 44, 131 (2001).

[21] J. Alicea, New directions in the pursuit of Majorana fermions in solid state systems, Rep. Prog. Phys. 75, 076501 (2012).

[22] S. Kezilebieke, M. Dvorak, T. Ojanen, and P. Liljeroth, Coupled Yu-Shiba-Rusinov states in molecular dimers on $\mathrm{NbSe}_{2}$, Nano Lett. 18, 2311 (2018).

[23] M. Assig, M. Etzkorn, A. Enders, W. Stiepany, C. R. Ast, and $\mathrm{K}$. Kern, A $10 \mathrm{mK}$ scanning tunneling microscope operating in ultra high vacuum and high magnetic fields, Rev. Sci. Instrum. 84, 033903 (2013).

[24] H. Huang, C. Padurariu, J. Senkpiel, R. Drost, A. L. Yeyati, J. C. Cuevas, B. Kubala, J. Ankerhold, K. Kern, and C. R. Ast, Tunnelling dynamics between superconducting bound states at the atomic limit, Nat. Phys. 16, 1227 (2020). 
[25] See Supplemental Material at http://link.aps.org/supplemental/ 10.1103/PhysRevResearch.3.L032008 for details on the experiment, theory and data analysis, which includes Refs. $[2,8,24,26,27,29,30,32-42,44-58]$.

[26] A. V. Balatsky, I. Vekhter, and J. X. Zhu, Impurity-induced states in conventional and unconventional superconductors, Rev. Mod. Phys. 78, 373 (2006).

[27] L. Cornils, A. Kamlapure, L. Zhou, S. Pradhan, A. Khajetoorians, J. Fransson, J. Wiebe, and R. Wiesendanger, Spin-Resolved Spectroscopy of the Yu-Shiba-Rusinov States of Individual Atoms, Phys. Rev. Lett. 119, 197002 (2017).

[28] L. Schneider, P. Beck, J. Wiebe, and R. Wiesendanger, Atomic-scale spin-polarization maps using functionalized superconducting probes, Sci. Adv. 7, eabd7302 (2021).

[29] A. Villas, R. L. Klees, H. Huang, C. R. Ast, G. Rastelli, W. Belzig, and J. C. Cuevas, Interplay between Yu-Shiba-Rusinov states and multiple Andreev reflections, Phys. Rev. B 101, 235445 (2020).

[30] M. Ruby, F. Pientka, Y. Peng, F. von Oppen, B. W. Heinrich, and K. J. Franke, Tunneling Processes into Localized Subgap States in Superconductors, Phys. Rev. Lett. 115, 087001 (2015).

[31] S. Loth, S. Baumann, C. P. Lutz, D. Eigler, and A. J. Heinrich, Bistability in atomic-scale antiferromagnets, Science 335, 196 (2012).

[32] C. R. Ast, B. Jack, J. Senkpiel, M. Eltschka, M. Etzkorn, J. Ankerhold, and K. Kern, Sensing the quantum limit in scanning tunnelling spectroscopy, Nat. Commun. 7, 13009 (2016).

[33] M. H. Devoret, D. Esteve, H. Grabert, G. L. Ingold, H. Pothier, and C. Urbina, Effect of the Electromagnetic Environment on the Coulomb Blockade in Ultrasmall Tunnel-Junctions, Phys. Rev. Lett. 64, 1824 (1990).

[34] D. V. Averin, Y. V. Nazarov, and A. A. Odintsov, Incoherent tunneling of the Cooper pairs and magnetic-flux quanta in ultrasmall Josephson-junctions, Physica B 165-166, 945 (1990).

[35] G. L. Ingold, H. Grabert, and U. Eberhardt, Cooper-pair current through ultrasmall Josephson-junctions, Phys. Rev. B 50, 395 (1994).

[36] F. Pientka, L. I. Glazman, and F. von Oppen, Topological superconducting phase in helical Shiba chains, Phys. Rev. B 88, 155420 (2013).

[37] F. Pientka, L. I. Glazman, and F. von Oppen, Unconventional topological phase transitions in helical Shiba chains, Phys. Rev. B 89, 180505(R) (2014).

[38] S. Hoffman, J. Klinovaja, and D. Loss, Topological phases of inhomogeneous superconductivity, Phys. Rev. B 93, 165418 (2016).

[39] G. M. Andolina and P. Simon, Topological properties of chains of magnetic impurities on a superconducting substrate: Interplay between the Shiba band and ferromagnetic wire limits, Phys. Rev. B 96, 235411 (2017).

[40] J. Klinovaja, P. Stano, A. Yazdani, and D. Loss, Topological Superconductivity and Majorana Fermions in RKKY Systems, Phys. Rev. Lett. 111, 186805 (2013).
[41] M. Vazifeh and M. Franz, Self-Organized Topological State with Majorana Fermions, Phys. Rev. Lett. 111, 206802 (2013).

[42] B. Braunecker and P. Simon, Interplay between Classical Magnetic Moments and Superconductivity in Quantum OneDimensional Conductors: Toward a Self-Sustained Topological Majorana Phase, Phys. Rev. Lett. 111, 147202 (2013).

[43] M. Eschrig, J. Kopu, J. C. Cuevas, and G. Schön, Theory of Half-Metal/Superconductor Heterostructures, Phys. Rev. Lett. 90, 137003 (2003).

[44] S. Sekula and R. Kernohan, Magnetic properties of superconducting vanadium, Phys. Rev. B 5, 904 (1972).

[45] J. Zasadzinski, D. Burnell, E. Wolf, and G. Arnold, Superconducting tunneling study of vanadium, Phys. Rev. B 25, 1622 (1982).

[46] V. Jensen, J. N. Andersen, H. B. Nielsen, and D. L. Adams, The surface-structure of V(100), Surf. Sci. 116, 66 (1982).

[47] R. Koller, W. Bergermayer, G. Kresse, E. L. D. Hebenstreit, C. Konvicka, M. Schmid, R. Podloucky, and P. Varga, The structure of the oxygen induced $(1 \times 5)$ reconstruction of $\mathrm{V}(100)$, Surf. Sci. 480, 11 (2001).

[48] F. Dulot, P. Turban, B. Kierren, J. Eugene, M. Alnot, and S. Andrieu, (001)V surface structures analysed by RHEED and STM, Surf. Sci. 473, 172 (2001).

[49] B. Jäck, M. Eltschka, M. Assig, M. Etzkorn, C. R. Ast, and K. Kern, Critical Josephson current in the dynamical Coulomb blockade regime, Phys. Rev. B 93, 020504(R) (2016).

[50] M. T. Randeria, B. E. Feldman, I. K. Drozdov, and A. Yazdani, Scanning Josephson spectroscopy on the atomic scale, Phys. Rev. B 93, 161115(R) (2016).

[51] J. C. Cuevas, A. Martín-Rodero, and A. L. Yeyati, Hamiltonian approach to the transport properties of superconducting quantum point contacts, Phys. Rev. B 54, 7366 (1996).

[52] J.-X. Zhu, Bogoliubov-de Gennes Method and its Applications (Springer, Heidelberg, 2016), Vol. 924.

[53] S. Nadj-Perge, I. K. Drozdov, B. A. Bernevig, and A. Yazdani, Proposal for realizing Majorana fermions in chains of magnetic atoms on a superconductor, Phys. Rev. B 88, 020407(R) (2013).

[54] J. Li, H. Chen, I. K. Drozdov, A. Yazdani, B. A. Bernevig, and A. H. MacDonald, Topological superconductivity induced by ferromagnetic metal chains, Phys. Rev. B 90, 235433 (2014).

[55] A. Heimes, P. Kotetes, and G. Schön, Majorana fermions from Shiba states in an antiferromagnetic chain on top of a superconductor, Phys. Rev. B 90, 060507(R) (2014).

[56] K. Pöyhönen, A. Westström, and T. Ojanen, Topological superconductivity in ferromagnetic atom chains beyond the deep-impurity regime, Phys. Rev. B 93, 014517 (2016).

[57] A. Theiler, K. Björnson, and A. M. Black-Schaffer, Majorana bound state localization and energy oscillations for magnetic impurity chains on conventional superconductors, Phys. Rev. B 100, 214504 (2019).

[58] B. Jäck, Y. Xie, J. Li, S. Jeon, B. A. Bernevig, and A. Yazdani, Observation of a Majorana zero mode in a topologically protected edge channel, Science 364, 1255 (2019). 